\title{
Bayesian Methods for Exoplanet Science
}

\author{
Hannu Parviainen
}

\begin{abstract}
Exoplanet research is carried out at the limits of the capabilities of current telescopes and instruments. The studied signals are weak, and often embedded in complex systematics from instrumental, telluric, and astrophysical sources. Combining repeated observations of periodic events, simultaneous observations with multiple telescopes, different observation techniques, and existing information from theory and prior research can help to disentangle the systematics from the planetary signals, and offers synergistic advantages over analysing observations separately. Bayesian inference provides a self-consistent statistical framework that addresses both the necessity for complex systematics models, and the need to combine prior information and heterogeneous observations. This chapter offers a brief introduction to Bayesian inference in the context of exoplanet research, with focus on time series analysis, and finishes with an overview of a set of freely available programming libraries.
\end{abstract}

\section{Introduction}

Statistical inference has a major role in the interpretation of astrophysical observations. Exoplanet research is generally carried out at the limits of the capabilities of current telescopes and instruments, and the planetary signals of interest are weak and embedded in complex systematics (noise) from instrumental, telluric, and astrophysical sources. The reliability of the information inferred from the observations depends on how well we understand the statistical characteristics of the observa-

Hannu Parviainen

Instituto de Astrofísica de Canarias, C. Via Lactea S/N, E-38205, La Laguna, Tenerife, Spain;

Dpto. de Astrofísica, Universidad de La Laguna, 38206,La Laguna, Tenerife, Spain; e-mail: hannuliac.es 
tions, and on how well these characteristics are taken into account by the methods used to carry out the inference.

Of the two major schools of statistical inference, frequentist and Bayesian, the latter has gained popularity in physics over the last decades. The reasons for the increasing interest in Bayesian methods are manifold: first, Bayesian inference offers a consistent approach for combining observational information from different types of observations (e.g., radial velocities, ground- and space-based photometric time series, etc.) with prior information; second, Bayesian inference allows for versatile modelling of the observational uncertainties (errors), facilitating robust analyses; and third, Bayesian inference offers an unified, self-consistent, approach for parameter estimation and model comparison. However, Bayesian inference is in general computationally more demanding than the frequentist approaches, and its wide adoption has been made possible only by the recent advances in numerical methods and computing power.

The Bayesian approach assigns a probability to a hypothesis. A prior probability distribution is used to encode our prior information about the hypothesis, and this prior is updated using observations to obtain a posterior probability distribution. When new data is acquired, the posterior based on the previous data can be used as a prior distribution, and the new data is used to obtain a new, updated, posterior distribution.

The problems in statistical inference can be roughly divided into parameter estimation and model comparison. Bayesian parameter estimation aims to infer the joint posterior distribution for model parameters given a model (that we believe is sufficient to explain the observations), prior information, and observations, while model comparison aims to find which of the possible models is best-suited to explain the observations.

Bayesian parameter estimation results in a joint posterior distribution for the model parameters. This offers improved versatility over frequentist methods that generally yield point estimates, since the ways to summarise the posterior can be tailored to take its complexity into account. For example, an approximately normal posterior can be summarised by its mean and central $68 \%$ posterior interval to yield the common mean and $1 \sigma$ uncertainty estimate; while a powerlaw-like posterior can be summarised by fitting an appropriate analytic distribution; and a more complex posterior can be described by a set of percentiles, or as a mixture of analytic distributions. A common case in exoplanet characterisation is to encounter parameters (eccentricity, impact parameter, etc.) where the posterior is close-to constant for small parameter values, and starts tapering off towards large values after some point. In these cases the data can only constrain the upper boundary for the parameter, and reporting a point estimate (other than the upper boundary corresponding to some posterior percentile) would make little sense.

In Bayesian model comparison setting we are usually either interested in finding out how many physical signals a dataset contains (e.g., radial velocity planet searches), or what is the most likely physical cause for a signal (statistical validation of planet candidates found by transit and RV surveys). Other use cases exists, but these two are currently the dominant uses for model comparison in exoplanet 
research. In the first case, we want to find out when to stop introducing complexity (hypothetical planets) to the model. We are not only interested in finding the model that best fits the given data, but we want to find the model that explains the actual information content in the data without overfitting (fitting the noise). In the latter case, the models can be of similar complexity (e.g., a planet candidate signal can be caused by a bona-fide planet or a blended eclipsing binary), but the model comparison needs to combine evidence from different data sources. For example, the probability that a transiting planet candidate is a real planet can be estimated by combining information from the discovery light curve (transit shape, secondary eclipse depth, phase variations, etc.), Milky Way population synthesis simulations (blending probability given the aperture size and host star location), stellar characterisation, ground-based follow-up observations, etc.

This chapter aims to offer an introduction to the use of Bayesian inference in exoplanet research, with a focus on time series analysis (photometry and radial velocity observations). We present the basic concepts, theory, and methods, and also overview some issues specific to exoplanet research. However, given the depth of the topic, the chapter can only scratch the surface of Bayesian inference, and does not discuss the more advanced topics. Instead, we aim to direct the reader to more in-depth books and publications thorough the chapter, and finish with an overview to useful literacy.

\section{Theory}

The goal of Bayesian inference is to make probability statements about unobserved quantities conditional on observed data (freely citing Gelman et al. 2013, Sect. 1.3). In the case of parameter estimation (model fitting), we want to infer the joint probability distribution for the model parameters given observational data and prior information. This probability distribution is called the posterior distribution (posterior), and it is obtained by updating a prior distribution (prior) with a sampling distribution (also known as the likelihood, or data distribution.)

We follow the notation laid out by Gelman et al. (2013, Sect. 1.2), where $\boldsymbol{\theta}$ is a vector containing the unobservable quantities (model parameters), $\boldsymbol{y}$ is a vector containing the observed data (e.g., RV measurements or photometry), and $\boldsymbol{X}$ is a matrix containing the explanatory variables (also known as covariates, independent variables, or predictors) for the observations. Both the model parameters and observations are modelled as random quantities, but the covariates are considered to be known exactly. In general, the elements of $\boldsymbol{y}$ can be vectors themselves, in which case we have a matrix of observations $\boldsymbol{Y}$. This is, however, not that usual in exoplanet research (transmission spectroscopy being an exception, where the observed dataset consists of a set of narrow-band light curves constructed from a spectroscopic time series), so we consider each $\boldsymbol{y}$ element as scalar for simplicity. The covariates-such as the mid-exposure time, airmass, and seeing, when dealing with ground-based observations-are stored as vectors in covariate matrix, $\boldsymbol{X}$, one per ob- 
servation. However, if we have only a single covariate (usually time in time series analysis), we represent the covariate vector as $\boldsymbol{x}$.

\section{Posterior distribution}

The posterior distribution encodes the information about the model parameters given the prior information and the likelihood from observations. Posterior probabilities for individual parameters are obtained through marginalisation, where the posterior is integrated over all other parameters than the parameter of interest. Finally, different summary statistics can be derived to describe the posterior distributions concisely.

The joint posterior distribution can be derived starting from the Bayes' theorem

$$
\operatorname{Pr}(H \mid D)=\frac{\operatorname{Pr}(H) \operatorname{Pr}(D \mid H)}{\operatorname{Pr}(D)},
$$

where $\operatorname{Pr}(H \mid D)$ is the posterior probability for a hypothesis $H$ given data $D, \operatorname{Pr}(H)$ is the prior probability for the hypothesis, $\operatorname{Pr}(D \mid H)$ is the probability for the data given the hypothesis, and $\operatorname{Pr}(D)$ is the probability for the data. In parameter estimation setting, the hypothesis $H$ is a (continuous) model parameter vector $\boldsymbol{\theta}$, the probabilities are continuous probability distributions, and the posterior distribution is

$$
P(\boldsymbol{\theta} \mid \boldsymbol{y})=\frac{P(\boldsymbol{\theta}) P(\boldsymbol{y} \mid \boldsymbol{\theta})}{P(\boldsymbol{y})}=\frac{P(\boldsymbol{\theta}) P(\boldsymbol{y} \mid \boldsymbol{\theta})}{\int P(\boldsymbol{\theta}) P(\boldsymbol{y}) \mathrm{d} \boldsymbol{\theta}} .
$$

The integral in the denominator, probability for the data, is a normalising constant called marginal probability (or model evidence), and ensures that the posterior integrates to unity.

\section{Prior distribution}

The role of a prior distribution is to encapsulate the current information and assumptions about a model parameter (or a quantity that depends on the model parameters). As new information (observations) is obtained, the prior is updated by the likelihood to produce a posterior distribution, which can be used as a prior distribution in future analyses.

Priors can be (roughly) classified as either informative priors or weakly informative (uninformative) priors, depending on how strongly they constrain the parameter space. Informative priors can be based on previous research and theory. For example, one can use a normal distribution with mean and standard deviation based on previously reported parameter mean and uncertainty estimates. Weakly informative 
priors are used to express our ignorance about a parameter, and aim to minimise the effect the prior has on the posterior, allowing the data to 'speak for itself'.

The choice of a prior distribution is not objective. When setting informative priors based on previous research, one needs to decide how much the reported values can be trusted. For example, if several competing estimates exists, it may be better to create a prior that encompasses all the estimates, rather than base a prior on any single estimate. In any case, it is important to report the priors used in an analysis, and, unless trivial, describe the justification for the priors (i.e., how and why the priors were chosen). It is also a good practice to test how sensitive the analysis is on the priors. Sensitivity analysis is especially important in a model selection setting, where the priors generally have a larger impact than in parameter estimation.

\section{Likelihood}

The likelihood represents the probability that the observations follow from a given model evaluated at a given point in the model parameter space. In parameter estimation setting, the model generally consists of a deterministic and a stochastic part. The deterministic part models the signals that can be modelled using a parametric model, and the stochastic part aims to explain the noise, that is, everything not explained by the parametric model.

\section{White noise}

If the observations can be explained by a parametric model with additive uncorrelated (white) noise, the joint likelihood is a product of independent likelihoods,

$$
P(\mathbf{d} \mid \boldsymbol{\theta})=\prod_{i} P\left(d_{i} \mid \boldsymbol{\theta}\right),
$$

where $\mathbf{d}$ is the dataset, $d_{i}$ are the individual observations and $\boldsymbol{\theta}$ is the model parameter vector. The product in Eq. 3 can easily lead to numerical under- or overflows, and it is common to work with $\log$ densities instead, so that

$$
\ln P(\mathbf{d} \mid \boldsymbol{\theta})=\sum_{i} \ln P\left(d_{i} \mid \boldsymbol{\theta}\right) .
$$

As an example, if the noise is normally distributed, a single observation $y_{i}$ follows

$$
y_{i} \sim \mathscr{N}\left(m\left(\boldsymbol{\theta}, \boldsymbol{x}_{i}\right), \sigma_{i}\right),
$$

where $\mathscr{N}$ stands for the normal distribution, $m$ is the parametric model (in this case also called the mean function), $\boldsymbol{\theta}$ are the model parameters, $\boldsymbol{x}$ is the vector of covariates for observation $i$, and $\sigma_{i}$ is the standard deviation (uncertainty) for observation $i$. The likelihood of a single observation is now 


$$
P\left(y_{i} \mid \boldsymbol{\theta}, \boldsymbol{x}_{i}, \sigma_{i}\right)=\frac{1}{\sigma_{i} \sqrt{2 \pi}} \exp -\frac{\left(y_{i}-m\left(\boldsymbol{\theta}, \boldsymbol{x}_{i}\right)\right)^{2}}{2 \sigma_{i}^{2}},
$$

and the log likelihood of a dataset consisting of $n$ observations is

$$
\ln P(\boldsymbol{y} \mid \boldsymbol{\theta}, \boldsymbol{X}, \boldsymbol{\sigma})=-\frac{1}{2}\left(n \ln 2 \pi+\sum_{i}^{n} \ln \sigma_{\mathrm{i}}^{2}+\sum_{i=1}^{n} \frac{r_{i}^{2}}{2 \sigma_{\mathrm{i}}^{2}}\right),
$$

where $r_{i}$ is the residual, $r_{i}=y_{i}-m\left(\boldsymbol{\theta}, \boldsymbol{x}_{i}\right)$.

\section{Correlated noise}

Unfortunately, observational noise is rarely white. The instrument, Earth's atmosphere, and different astrophysical processes all give rise to signals, of which only a part can be represented as simple functions of quantities measured simultaneously with the observations (covariates). The rest of these noise signals aggregate into time-correlated (red) noise that has to be accounted for in the analysis using statistical methods. If not accounted for, red noise can lead to biased parameter estimates with underestimated uncertainties, and false detections in planet searches. For example, stellar granulation can lead to time-correlated photometric variability with amplitude and time-scale comparable to planetary transits, while star spots give rise to RV signals that can be mistaken as of planetary origin.

Correlated noise can be represented as a stochastic (random) process in time, and if the noise process follows a normal distribution, it can generally be modelled as a Gaussian process (GP, Rasmussen and Williams 2006, Roberts et al. 2013, Gibson et al. 2012). Generic Gaussian processes require an inversion of an $n \times n$ covariance matrix, where $n$ is the number of time series datapoints, which does not scale well for large time series. However, methods have been developed to evaluate temporally correlated GPs with better scaling, which allow GPs to be used in most time series analysis problems encountered in exoplanet research.

Generic Gaussian Processes The scalar log-likelihood Eq. 7 can be written in a more general vector form as

$$
\ln P(\boldsymbol{y} \mid \boldsymbol{\theta})=-\frac{1}{2}\left(n \ln 2 \pi+\ln |\boldsymbol{\Sigma}|+\mathbf{r}^{\mathrm{T}} \boldsymbol{\Sigma}^{-1} \mathbf{r}\right),
$$

where $n$ is the number of datapoints, $\mathbf{r}$ is the residual vector, and $\boldsymbol{\Sigma}$ is the covariance matrix. The covariance matrix is diagonal for white noise, which yields Eq. 7 as a special case, but contains off-diagonal elements when the noise is correlated.

Gaussian processes (GPs) offer a versatile way to model normally distributed stochastic processes, with extensions existing for Student-t-distributed processes (Shah et al.2014). In GP formalism, the covariance matrix elements are given by

$$
\boldsymbol{\Sigma}_{i, j}=k\left(\mathbf{x}_{i}, \mathbf{x}_{j}\right)+\sigma^{2} \delta_{i j},
$$


where $k$ is called the covariance function (or kernel), $\mathbf{x}$ are input parameter vectors, $\delta$ is the Kronecker delta function, and $\sigma^{2}$ is the white noise term for the $i$ th datapoint. The covariance function maps the input vectors to a scalar covariance, and thus defines the behaviour of the GP.

Gaussian processes are versatile: they can be used with any number of inputs and outputs; they can be used to model processes that combine aperiodic, periodic and quasi-periodic behaviour; and they can be used to extract different additive processes from a time series. However, in its most general form, a GP evaluation requires the inversion of a covariance matrix, which has a time complexity $\mathscr{O}\left(n^{3}\right)$, where $n$ is the number of input points.

Despite the bad scaling properties, GPs have been used in exoplanet research extensively. For example, Gibson et al. (2012) introduced Gaussian processes to the exoplanet research community as a robust tool to model systematics in transmission spectroscopy (with an excellent overview of the GP basics in the Appendix); (Rajpaul et al.2015) demonstrated how GPs can be used to disentangle the stellar activity signal from planetary signal(s) in radial velocity time series; and (Czekala et al.2017) showed how GPs can be used to model stellar spectra to improve radial velocity measurements.

The $\mathscr{O}\left(n^{3}\right)$ scaling restricts the use of generic GPs to problems with a relatively small number of datapoints. However, the matrix inversion can be accelerated (or bypassed completely) for several special cases, which are discussed below.

Temporally correlated noise with power law power spectral density Carter and Winn (2009) introduce a wavelet-based method to calculate the likelihood for a time series having temporally correlated noise with power spectral density (PSD) varying as $1 / f^{\gamma}$. The approach uses fast wavelet transform (FWT) and computes the likelihood in linear time (that is, the method has time complexity $\mathscr{O}(n)$ ). The method is implemented in many transit modelling packages, and offers a good alternative to the computation-heavy GPs if the time sampling is approximately uniform, time is used as the only input parameter, and if the noise PSD follows a power law. However, more versatile methods should be used if any of these restrictions is not met.

Temporally correlated noise with a (nearly) arbitrary kernel The computational cost of Gaussian processes can be alleviated in the special case when time is the only input parameter, and the kernel yields covariance matrices satisfying special conditions. (Ambikasaran et al.2014) describe an approach that allows for the covariance matrix inversion in $\mathscr{O}\left(n \log ^{2} n\right)$ time, and covariance matrix determinant computation in $\mathscr{O}(n \log n)$ time. This difference in scalability allows the approach to be used in the analysis of large time series, such as with Kepler light curves.

Temporally correlated noise with kernel consisting of complex exponentials Describing the covariance function as a mixture of complex exponentials allows one-dimensional GPs to be evaluated in linear time (Foreman-Mackey et al.|2017). Despite its restrictions, such a kernel is flexible enough to model a wide range of 
astrophysical variability, and is especially well-suited to model quasiperiodic behaviour.

\section{Marginal likelihood}

Marginal likelihood, also known as model evidence, can be ignored in parameter estimation setting, but is an important quantity in Bayesian model comparison. The marginal likelihood, $Z$, is obtained by integrating the posterior over the whole parameter space

$$
Z=\int P(\boldsymbol{\theta}) P(\boldsymbol{y} \mid \boldsymbol{\theta}) \mathrm{d} \boldsymbol{\theta} .
$$

The integration is rarely analytically tractable, and generally requires specialised numerical methods.

\section{Parameter estimation}

The aim of model parameter estimation is to obtain an estimate of the joint posterior distribution for the model parameters given the data and prior information. Per-parameter marginal posteriors can then be derived from the joint posterior, and reported concisely using different summarisation methods.

\section{Marginal posterior distribution}

The marginal likelihood can be ignored in the parameter estimation setting, allowing us to work with an unnormalised joint posterior density

$$
P(\boldsymbol{\theta} \mid \boldsymbol{y})=P(\boldsymbol{\theta}) P(\boldsymbol{y} \mid \boldsymbol{\theta}) .
$$

The posterior distribution for a single parameter $\theta_{i}$ is obtained by integrating the joint posterior density over all other parameters

$$
P\left(\theta_{i} \mid \boldsymbol{y}\right)=\int P(\boldsymbol{\theta} \mid \boldsymbol{y}) \mathrm{d} \boldsymbol{\theta}_{j \neq i} .
$$

This is called marginalisation, and results in a marginal posterior distribution for the parameter. 


\section{Summarising posteriors}

Bayesian inference is generally based on a random sample drawn from the posterior, which can then be described concisely using summary statistics. Common point estimates, such as the mean and the mode (or modes, if the posterior is multimodal), and a set of posterior percentiles all convey useful information. Especially, Gelman et al. (2013, see Sects. 2.3 and 10.5) recommend reporting the 2.5\%, 25\%, 50\%, $75 \%$, and $97.5 \%$ percentiles, which provide the posterior median, $50 \%$ and $95 \%$ posterior intervals, and also information about the posterior skewness.

If a marginal posterior can be approximately modelled by an analytical distribution, then the parameters of the distribution fitted to the samples can be reported. However, even then, it is useful to report the posterior percentiles to allow for easy comparison with other studies.

When practical, it is useful to visualise the marginal posteriors and joint pairwise posteriors. This can be done directly from the samples using either 1D and 2D histograms or kernel density estimation (KDE), and provides insight into the parameter distributions, and whether there were any problems in the sampling process.

\section{Estimating posteriors: Markov chain Monte Carlo}

Markov chain Monte Carlo (MCMC) sampling is a fundamental tool for posterior estimation when we cannot sample from the posterior directly. The method produces a set of samples drawn from the posterior by iteratively constructing a Markov chain with the posterior distribution as its equilibrium distribution.

The sampler starts a chain from a point in the parameter space, proposes a move to another point, and either accepts or rejects the move based on the posterior density ratios between the current and proposed locations. The location after the proposal is added to the chain (no matter whether the proposal was accepted or rejected), and the sampling continues by proposing a new step. The proposal move is constructed in a way to satisfy a set of conditions that ensure that the distribution of the samples in the chain asymptotically converges to the posterior distribution, but the speed of convergence depends on the complexity of the posterior and the MCMC implementation (see Gelman et al. 2013, Sect. 11; Robert 2007. Sect. 6.3; and Gregory 2005, Sect. 12 for a comprehensive explanation; and Betancourt 2017 for a historical overview).

General considerations Markov chain Monte Carlo is, actually, an umbrella term for a family of sampling methods. The basic MCMC approaches, such as the Metropolis-Hastings algorithm (Hastings 1970), are easy to code, but the more advanced methods, such as NUTS (Hoffman and Gelman 2011), are not straightforward to implement by oneself. MCMC sampling packages exist for all the major programming environments, some of which are listed in Sect. , and usually the first step to do when starting an analysis is to choose the best sampler applicable to 
the problem at hand. A good sampler can explore the posterior space efficiently, with a minimal number of posterior evaluations. The modern MCMC samplers, like NUTS, can be very efficient if the posterior derivatives can be calculated analytically. However, if this is not possible, a less-advanced sampler needs to be used.

While the different sampling approaches have their own peculiarities, some basic steps for obtaining a reliable posterior sample can be considered common:

1. Try to set up the problem in a way that allows for efficient sampling. For example, choose a sampling parametrisation that reduces mutual correlations between parameters, as discussed in Sect. . Bad model parametrisation can make it impossible for a sampler to explore the whole posterior space.

2. Test the sampler by simulating a set of short chains and check the acceptance rate and parameter autocorrelation lengths. See if the sampler has parameters that can be tuned to improve the sampling, or try to reparametrise the problem if the sampler is not able to explore the posterior properly. Modern MCMC packages also often offer an automatic tuning functionality, which aims to optimise the sampling.

3. Run multiple chains starting from different locations in the parameter space. Simulating multiple chains allows one to test for chain convergence, and can reveal posterior multimodality.

4. Test for chain convergence using numerical convergence tests. Most MCMC packages implement several convergence tests. If the chains have not converged, continue sampling.

5. Inspect the chains and parameter posteriors visually. This may not always be practical, e.g., if the MCMC simulations need to be repeated for many targets, but visual inspection may give useful insight about the convergence, sampling, and warm-up phase.

Acceptance rate The fraction of accepted jump proposals to the number of total proposals (acceptance rate) is an useful diagnostic of the sampler's efficiency. For multidimensional models, the acceptance rate should be close to $23 \%$ (Gelman et al. 1996; Roberts et al.(1997).

Chain warm-up With a finite chain, the starting location can affect the chain's convergence. For example, if starting the chain far from the posterior mode, it may take time before the chain starts sampling the high-probability posterior space. The influence of the starting point is reduced by excluding an initial warm-up (or burnin) period (Gelman et al. 2013, p. 282). There are no hard rules for deciding the appropriate length for the warm-up period, but it generally can be decided based on convergence tests and visual inspection of chains.

Autocorrelation and thinning The consecutive samples in the MCMC chain are not independent, but correlated, and the strength of correlation depends on the sampler's ability to sample the posterior. A set of independent samples can be obtained by thinning the chain, i.e., by selecting every $n$th sample, where $n$ is close to the chain autocorrelation length. However, chain thinning is not strictly necessary if the 
chains have converged (Link and Eaton|2012; Gelman et al. 2013, p. 282), but can still be practical for memory and disk-space saving purposes.

Testing chain convergence The MCMC chain approximates the posterior asymptotically. However, the number of steps needed to obtain a representative sample depends on how efficiently the sampler can cover the posterior, and the chains need to be tested for convergence before they can be used for inference. Converged chains should be statistically similar to each other, and thus a set of chains started from different points in the parameter space allows us to test for convergence of the chains in the set. The tests can be further improved by splitting the individual chains into two parts (after removing the warm-up phase), since the halves should be similar to each other if the chains are stationary and well-mixed.

The Gelman-Rubin diagnostic (Gelman and Rubin 1992, also, Gelman et al. 2013, p. 284) offers a practical way to test convergence using a set of chains. The diagnostic compares the estimate for the marginal posterior variance $V$ for parameter $\theta$ to the mean within-chain variance $W$. The two should be approximately equal for a set of well-converged chains, and the estimated scale reduction for $M$ chains with $N$ steps,

$$
\sqrt{\hat{R}}=\sqrt{\frac{N-1}{N}+\frac{M+1}{N M} \frac{B}{W}},
$$

should be close to unity (we have dropped the factor $\mathrm{df} /(\mathrm{df}-2)$ from Gelman and Rubin 1992, Eq. 20). Here $B$ is the between-chain variance, and $W$ the within-chain variance,

$$
\begin{aligned}
B & =\frac{N}{M-1} \sum_{m=1}^{M}\left(\hat{\theta}_{m}-\hat{\theta}\right)^{2}, \\
W & =\frac{1}{M} \sum_{m=1}^{M} \hat{\sigma}_{m}^{2}
\end{aligned}
$$

where $\hat{\theta}$ is the parameter mean estimated using all samples, $\hat{\theta}_{m}$ is the mean estimated from a single chain, and $\hat{\sigma}_{m}^{2}$ is the parameter variance estimated from a single chain.

\section{Model comparison}

Bayesian model comparison is based on model evidence (also known as marginal likelihood)

$$
Z=\int P(\boldsymbol{\theta}) P(\boldsymbol{y} \mid \boldsymbol{\theta}) \mathrm{d} \boldsymbol{\theta},
$$

which we also recognise as the normalising constant in Eq. 2, i.e., the integrated posterior density. Here, the multidimensional integration becomes the main problem. The integration can rarely be carried out analytically and numerical integration of multidimensional densities is far from trivial. 
Table 1 Guidelines for interpreting Bayes factors, as presented by Kass and Raftery (1995).

\begin{tabular}{lll}
\hline \hline $2 \ln B_{10}$ & $B_{10}$ & Evidence against $H_{0}$ \\
\hline 0 to 2 & 1 to 3 & Not worth more than a bare mention \\
2 to 6 & 3 to 20 & Positive \\
6 to 10 & 20 to 150 & Strong \\
$>10$ & $>150$ & Very strong \\
\hline
\end{tabular}

The use of model evidence in model comparison aims to penalise model complexity that is not justified by the data: since the posterior is the likelihood multiplied by the prior, and since each additional model parameter must be accompanied by a prior reflecting our knowledge about the parameter, the addition of a model parameter must increase the likelihood more than the prior penalises the posterior (Gregory 2005, Sect. 3.5; MacKay 2003, Sect. 28.1).

Given two competing models, the posterior odds in favour of model $M_{1}$ over model $M_{2}$ are

$$
O_{12}=\frac{\operatorname{Pr}\left(M_{1} \mid \boldsymbol{y}\right)}{\operatorname{Pr}\left(M_{2} \mid \boldsymbol{y}\right)}=\frac{Z_{1}}{Z_{2}} \frac{P\left(M_{1}\right)}{P\left(M_{2}\right)}=B_{12} \frac{P\left(M_{1}\right)}{P\left(M_{2}\right)},
$$

where $P\left(M_{1}\right)$ and $P\left(M_{2}\right)$ are the prior model probabilities and $B_{12}$ is called the Bayes factor, the ratio of the model evidences. Given a set of models, the posterior model probabilities can be calculated from the posterior odds as

$$
P\left(M_{i} \mid \boldsymbol{y}\right)=\frac{O_{i 1}}{\sum_{1}^{n} O_{i 1}},
$$

but model comparison is usually carried out based on odds or log-odds. If the models have equal prior probabilities, the prior ratio equals to unity, and the model comparison can be done purely based on the Bayes factors.

Kass and Raftery (1995) provide general guidelines for interpreting Bayes factors or odds ratios, reproduced in Table 1. The limits are somewhat arbitrary, and should not be taken as hard rules. Bayesian evidence can be very sensitive on the choice of priors, and sensitivity analysis should be carried out as a standard part of the analysis.

Methods for evidence estimation start from simple approaches that can work with low-dimensional models, such as direct Monte Carlo integration; to slightly more involved, such as different importance sampling approaches; to complicated, such as the more advanced nested sampling approaches, bridge and path sampling, and Bayesian quadrature. The methods are too numerous and intricate to be presented here, but are thoroughly reviewed by Clyde et al. (2007), Ford et al. (2007), and Robert (2007, Sect. 7).

Nested sampling (Skilling 2004, 2006, Chopin and Robert 2010) has gained popularity in astrophysics. The method provides both an evidence estimate and a sample from the posterior, and has publicly available implementations that can efficiently 
sample posteriors with multiple modes (Feroz et al. 2009, 2013) and phase transitions (Brewer 2014). The software packages are listed in Sect. .

Finally, Bayesian quadrature (Rasmussen and Ghahramani 2002, Osborne et al. 2012: Gunter et al. 2014; Hennig et al. 2015) offers an integration approach that can be useful with relatively low-dimensional problems where the posterior evaluation is computationally expensive. Bayesian quadrature approximates the posterior density as a Gaussian process, which can be integrated analytically, and allows for intelligent sampling.

As a final note, the Bayesian community is somewhat divided what comes to model comparison and the use of Bayesian evidence. Model comparison problems can often be transformed into parameter estimation problems, in which case a parameter estimation approach can advisable (Gelman et al.2013, Sect. 7.4).

\section{Practical issues in exoplanet research}

While the theory behind Bayesian inference is straightforward, obtaining a reliable posterior estimate can be tricky in practise. MCMC samplers can fail to properly sample multimodal posteriors, and the samplers can be inefficient if any of the parameters are strongly correlated. The optimal parametrisation for RV and transit modelling, and how to deal with the stellar limb darkening in transit modelling, are especially important practical issues in exoplanet research, and we briefly outline the current best practices below.

\section{Multimodal posteriors}

Multimodal posteriors are rare in transit light curve modelling (indeed, a multimodal posterior in transit analysis most often means that there is a problem in the code or the data), but quite common in radial velocity planet searches.

The main issue with multimodal posteriors is that an MCMC sampler can fail to travel through the valleys between the modes, and get stuck to sample the local mode nearest to where the chain was started from. In this case, the multimodality might not be visible from the posterior estimate at all, and we might end up with an overly simplistic picture of the posterior space. This can be alleviated by

- starting multiple chains from random locations in the parameter space (for example, drawing the starting locations from the prior),

- starting multiple chains close to the posterior maxima obtained using a global optimiser,

- starting multiple chains close to the maxima estimated using local optimiser started from different points in the parameter space,

- or using an MCMC approach designed to sample multimodal distributions efficiently (such as parallel tempering MCMC). 


\section{Parametrisation in transit and $R V$ modelling}

Basic MCMC algorithms, such as Metropolis-Hastings, are most efficient sampling approximately multivariate normal posteriors with minimal correlation between parameters (this is because the proposal distribution is usually multivariate normal). Inefficient sampling shows as a long autocorrelation time, and means that a long chain is required to obtain a representative posterior sample. It generally makes sense to carry out the sampling with a parameter set that aims to minimise mutual correlations (sampling parametrisation), which is then mapped to the native model parametrisation.

Priors must be considered when deciding the sampling parameters. A weakly informative (noninformative) prior on a sampling parameter will not be a weakly informative prior on the model parameter if the mapping from one to another involves nonlinear transformations (Burke et al.2007, see the appendix). For example, a uniform prior on planet-star area ratio, $d=k^{2}$, where $k$ is the planet-star radius ratio, does not lead to a uniform prior on the radius ratio. While this is not a significant issue with parameters strongly constrained by the likelihood, it may lead to biases for parameters for which the observations do not yield a lot of information (that is, when the prior dominates the posterior). If one desires to set a weakly informative prior on a model parameter that is mapped from the sampling parametrisation through a nonlinear transformation, one needs to calculate the Jacobian of the transformation.

Ford (2006); Carter et al. (2008); Kipping (2010) (among others) have investigated how the sampling parameter set affects the efficiency of basic MCMC routines in the RV and transit light curve modelling. A transit model can be generally defined using from 7 to 11 parameters, and an RV model with five parameters. Typical "physical" model parametrisations are

Transit model: 1) zero epoch; 2) orbital period; 3) orbital inclination; 4) semimajor axis; 5) eccentricity; 6) argument of periastron; 7) planet-star radius ratio; and 8) $n$ limb darkening coefficients, depending on the limb darkening model

Radial velocity model: 1) zero epoch; 2) orbital period; 3) eccentricity; 4) argument of periastron; and 5) $M \sin i$

but the optimal sampling parametrisation depends on the purpose of the analysis. Below we consider generic transit or RV modelling, but, for example, RV planet searches can benefit from using $\log p$ as a sampling parameter, where $p$ is the orbital period, instead of $p$.

Inclination and impact parameter In transit light curve modelling, orbital inclination $i$ can be substituted by the impact parameter $b$. The mapping is

$$
b=a_{s} \cos i\left[\frac{1-e^{2}}{1+e \sin \omega}\right], \quad i=\arccos \left(\frac{b}{a_{s}}\left[\frac{1+e \sin \omega}{1-e^{2}}\right]\right) \text {, }
$$

where $a_{s}=a / R_{\star}$, that is, the semi-major axis divided by the stellar radius, $e$ is the eccentricity and $\omega$ the argument of periastron. The eccentricity-dependent term in square brackets equals to unity for circular orbits, and can be ignored for small $e$. 
The impact parameter is bounded by $[0,1+k]$ for a transiting planet, where $k$ is the planet-star radius ratio.

Semi-major axis and stellar density The scaled semi-major axis, $a_{s}$, can be replaced by transit duration (Kipping 2010) or stellar density, $\rho_{\star}$. The latter is practical since information from stellar characterisation can be used to create physicallybased informative priors on stellar density, and the estimated stellar density can be used in planet candidate validation (Seager and Mallen-Ornelas 2003, Tingley et al. 2011; Kipping 2014). The mapping can be derived from the Kepler's third law, and is

$$
a_{s}=\left(\frac{G \rho_{\star} p^{2}}{3 \pi}\right)^{1 / 3}, \quad \rho_{\star}=\frac{3 \pi a_{s}^{3}}{G p^{2}}
$$

where $G$ is the gravitational constant, $p$ is the period, and $\rho$ is the stellar density (all in SI units).

Eccentricity and argument of periastron Direct sampling in eccentricity, $e$, and argument of periastron, $\omega$, can be inefficient with most MCMC samplers. The argument of periastron is not well constrained for low-eccentricity orbits, and it can have a bimodal posterior if modelling only photometry without RV observations (such as when modelling light curves observed over the whole orbital phase, where the phase curve and secondary eclipse yield information about $e$ and $\omega$ ).

Ford (2005) suggested using $e \cos \omega$ and $e \sin \omega$ as an efficient sampling parametrisation, but noted later that setting an uniform prior on the parameters would set a non-uniform prior on the eccentricity, leading to biased eccentricity estimates (Ford 2006). Anderson et al. (2011) improved the parametrisation slightly into

$$
a=\sqrt{e} \cos \omega, \quad b=\sqrt{e} \sin \omega,
$$

which can be mapped to $e$ and $\omega$ as

$$
e=a^{2}+b^{2}, \quad \omega=\arctan (b, a) .
$$

This parametrisation ensures that an uniform prior on $a$ and $b$ leads to an uniform prior on the eccentricity.

\section{Stellar limb darkening in transit modelling}

Stellar limb darkening (LD) is a significant source of uncertainty in transit modelling. The transit codes represent the stellar limb darkening profile as a linear combination of basis functions (Mandel and Agol 2002; Giménez 2006). The coefficients of these functions (limb darkening coefficients, LDC) are mutually correlated and degenerate with the planet-star radius ratio and impact parameter. That is, a wide combination of LDC values, radius ratios and impact parameters may explain the observations equally well. 
Several approaches have been used in attempt to overcome the degeneracies: the LDC can be fixed to theoretical values, they can be left completely unconstrained in order to marginalise over the whole LDC space allowed by the data, or they can be constrained with informative priors based on theoretical models.

Fixed limb darkening coefficients In the early phases of transiting exoplanet research, the limb darkening coefficients were usually fixed to values interpolated from limb darkening coefficient tables based on numerical stellar models, such as the tables by Claret (2004); Claret and Bloemen (2011); Claret et al. (2012, 2014) and Sing (2010). This is an easy way to circumvent the complications due to limb darkening if the stellar models are reliable. However, if they are not (they were not, see Claret 2008, 2009), fixing the limb darkening coefficients leads to biased parameter estimates (Csizmadia et al. 2013, Espinoza and Jordan 2015, but see also Müller et al.|2013 for a counterargument).

The stellar models have improved during the last decade, and the current models can be considered more reliable than the ones compared by Claret (2008, 2009). However, the possible biases are not the only problem when fixing the LDCs. Even in the case of a perfect stellar model, we generally do not know the planet host star perfectly. Fixing the LDCs does not allow us to account for the uncertainties in the stellar parameters, which leads to underestimated uncertainties in the planet characterisation.

Thus, fixing the LDC cannot really be advocated in a Bayesian setting, since it can lead to biases and ignores the uncertainties in the stellar characterisation.

Unconstrained limb darkening coefficients A second approach is to leave limb darkening completely unconstrained, so that the radius ratio and impact parameter estimates are marginalised over the whole limb darkening parameter space allowed by the data. This is the best way to minimise the parameter estimate biases, but since the LD coefficients are mutually correlated, the approach can reduce the MCMC sampling efficiency.

Here again, the sampling efficiency can be improved by using a less correlated parametrisation. Using the quadratic limb darkening model as an example, linear combinations of the two coefficients have often been used as sampling parameters (e.g. Holman et al. 2006, Carter et al. 2009). Especially, a mapping proposed by Kipping (2013)

$$
\begin{aligned}
u_{1}=2 \sqrt{q_{1}} q_{2}, & u_{2}=\sqrt{q_{1}}\left(1-2 q_{2}\right), \\
q_{1}=\left(u_{1}+u_{2}\right)^{2}, & q_{2}=\frac{u_{1}}{2\left(u_{1}+u_{2}\right)},
\end{aligned}
$$

where $u_{1}$ and $u_{2}$ are the quadratic coefficients, and $q_{1}$ and $q_{2}$ are the sampling parameters, has proven practical. With most parametrisations, one needs to carry out separate tests to ensure that the coefficients yield physically viable limb darkening profiles, but this mapping allows one to use uniform priors from 0 to 1 on $q_{1}$ and $q_{2}$ to cover the whole physically viable $\left(u_{1}, u_{2}\right)$-space. 
Kipping (2016) introduced a similar mapping for the three-parameter non-linear limb darkening model by Sing et al. (2009), and provides code implementing the mapping, its inverse, and the analytical criteria for the physical validity of the threeparameter LD model from https: //github. com/davidkipping/LDC3.

Limb darkening coefficients constrained by model-based informative priors The third approach is a compromise between the two extremes. The LD coefficients can be assigned informative priors based on stellar atmosphere models and the uncertainties in the host star characterisation. This approach allows one to use information from the models, but reduces biases and is less likely to seriously underestimate the parameter uncertainties. While the creation of priors is slightly more complicated than either of the two other approaches, tools exist to make the approach straightforward (Parviainen and Aigrain 2015; Espinoza and Jordan|2015).

\section{Tools for Bayesian inference}

We finish the chapter with a list of some of the most matured tools for posterior estimation, nested sampling, and Gaussian processes that provide a PYTHON interface. (We constrain the discussion to PYTHON since it is currently the de facto programming language for astrophysics. All major programming languages have similar packages available.)

Posterior estimation Several generic packages exist for posterior estimation using MCMC sampling.

PYMC3 by Salvatier et al. (2015) offers a versatile toolkit for Bayesian inference built on THEANO. The package includes a set of MCMC samplers, a Gaussian-process framework, and tools for working with more advanced topics, such as mixture models and variational inference. The package is available from https://github.com/pymc-devs/pymc3, and includes extensive documentation with tutorials and examples as IPython notebooks, which makes it very useful for learning Bayesian inference in practise.

STAN (http://mc-stan.org) is a probabilistic programming language that offers similar functionality as PYMC3, but with interfaces for many programming languages. For example, PYSTAN (https://github. com/stan-dev/pystan) provides an interface for PYTHON, and RSTAN (https://github.com/stan-dev/rstan) for R.

EDWARD is a Python library for probabilistic modelling (Tran et al.2016) built on TENSORFLOW, a machine learning framework developed by Google. EDWARD fuses three fields: Bayesian statistics and machine learning, deep learning, and probabilistic programming. http://edwardlib.org

EMCEE by Foreman-Mackey et al. (2013, https://github.com/dfm/ emcee) is a popular MCMC sampler implementing the affine-invariant ensemble sampler by Goodman and Weare (2010). The sampler is efficient 
in sampling correlated parameter spaces, and does not require the ability to calculate posterior derivatives, unlike many of the advanced MCMC samplers. The implementation also offers straightforward MPI parallelisation, which allows the sampling to be carried out efficiently in a cluster environment.

Nested sampling Of all the evidence estimation methods, nested sampling is currently the most popular (and accessible) one used in astrophysics. Nested sampling covers a variety of sampling methods, each with their advantages and disadvantages, and the research of new approaches is vibrant.

MULTINEST implements a nested sampling algorithm written in FORTRAN 90 that excels in sampling multimodal densities with strong degeneracies (Feroz et al.|2009, 2013). MultiNest draws samples from a constrained prior distribution approximated with ellipsoids, which makes it efficient for moderate dimensional problems (up to $\sim 40$ ), but other approaches can work better for high-dimensional problems (Brewer and Foreman-Mackey 2016). The code with $\mathrm{C} / \mathrm{C}++$ wrappers is available from Www.mrao. cam.ac.uk/software/multinest, and a PYTHON wrapper, PYMuLTiNest, is available from/https://github.com/JohannesBluchner/ PyMultiNest.

DNEST aims to sample multimodal distributions with degeneracies between parameters efficiently, like MultiNest, but uses MCMC for sampling (Brewer 2014, Brewer and Foreman-Mackey 2016). The code is written in C++, supports PYTHON and JULIA, and is available from https: //github.com/eggplantbren/DNest4.

NESTLE provides a pure-PYTHON implementation of nested sampling using either MCMC sampling, single-ellipsoid sampling, or MultiNest-like multi-ellipsoid sampling. The code is available from https://github. $\mathrm{com} / \mathrm{kbarbary} / \mathrm{nestle}$.

Gaussian processes In their most basic form, Gaussian processes (GPs) can be implemented with 3-5 lines of PYTHON code using SCIPY's linear algebra module. However, more advanced functionality-such as the ability to construct complex kernels, combine kernels, and solve the GP with better than $\mathscr{O}\left(n^{3}\right)$ scaling-requires more involved approaches. Gaussian processes are widely used by the machine learning community, and are usually implemented in one form or another in various machine learning packages.

GEORGE is a Gaussian process framework that implements two GP solvers: a basic solver that uses SCIPY's Cholesky decomposition, and a HODLR solver implementing the Ambikasaran et al. (2014) approach that scales as $\mathscr{O}\left(n \log ^{2} n\right)$. The package has been used widely by the astrophysics community, and is available from https://github.com/dfm/george.

CELERITE implements the $\mathscr{O}(n)$ GP solver described by Foreman-Mackey et al. (2017); Ambikasaran (2015). The approach is restricted to 1D processes where the covariance kernel is represented as a sum of complex expo- 
nentials, but is flexible enough to model a wide variety of astrophysical variability. The code with $\mathrm{C}++$ and PYTHON interfaces is available from https://github.com/dfm/celerite, and a JuLIA implementation can be found from https://github.com/ericagol/ celerite.jl.

GEePEA is a general GP package developed by Neale Gibson (Gibson et al.|2012, https://github.com/nealegibson/GeePea). The package is lightweight and easily extensible, and has been widely used in exoplanet transmission spectroscopy.

GPY is a Python GP framework developed by the Sheffield machine learning group. The package offers a wide variety of kernels, and is available from https://github.com/SheffieldML/GPy

GPFlow is a GP package using TensorFlow (Matthews et al. 2016). The package has been developed following GPY, but uses the TENSORFLOW machine learning framework to allow for faster and bigger computations. The package can be found from https://github.com/GPflow/ GPflow.

PYMC3 offers a lightweight but versatile GP framework with a set of basic covariance functions, and provides tools to modify the covariance matrix directly.

SCIKIT-LEARN is a multi-purpose machine learning package that includes Gaussian processes. The package is available from https://github.com/ scikit-learn/scikit-learn.

\section{Literature and resources}

Bayesian inference is a wide-ranging topic with books devoted to various subfields. This chapter has scratched the surface in the hope this will allow the reader to understand the basic concepts commonly encountered in Exoplanet research, but has not tried to delve into any of the more advanced topics, such as hierarchical modelling. However, in order to apply Bayesian methods in research, one should read at least one of the entry-level books dedicated to Bayesian inference

- Gregory (2005) covers the basics of Bayesian inference in an astrophysical context, with several exoplanet-related examples.

- Gelman et al. (2013) offers an overarching treatise to Bayesian inference, starting from the basics, but also covering a wide variety of advanced topics.

- Robert (2007) offers an in-depth look into Bayesian inference from the perspective of decision theory.

- MacKay (2003) covers Bayesian inference (amongst other topics) from the perspective of information theory.

Numerical methods improve continuously, and the landscape of generally accessible computational tools changes quickly. Internet is an important source of 
up-to-date information. Websites, such as Cross validated https://stats. stackexchange.com (choose tag "bayesian"), offer a way to connect researches not specialised to statistics with specialists, and statistics blogs, such as http: // andrewgelman.com and https://xianblog.wordpress.com, can help to keep one up-to-date with current developments in the field.

\section{References}

Ambikasaran S (2015) Generalized Rybicki Press algorithm. Numerical Linear Algebra with Applications 22(6):1102-1114, URL http://doi.wiley.com/10.1002/nla.2003

Ambikasaran S, Foreman-Mackey D, Greengard L, Hogg DW O’Neil M (2014) Fast Direct Methods for Gaussian Processes and the Analysis of NASA Kepler Mission Data URL http: //arxiv.org/abs/1403.6015

Anderson DR, Collier Cameron A, Hellier C et al. (2011) WASP-30b: A 61 M Jup BROWN DWARF TRANSITING A V = 12, F8 STAR. The Astrophysical Journal 726(2):L19, URL http://adsabs.harvard.edu/abs/2011ApJ...726L..19A

Betancourt M (2017) The Convergence of Markov chain Monte Carlo Methods: From the Metropolis method to Hamiltonian Monte Carlo URL http://arxiv.org/abs/1706.01520

Brewer BJ (2014) Inference for Trans-dimensional Bayesian Models with Diffusive Nested Sampling URL http://arxiv.org/abs/1411.3921

Brewer BJ Foreman-Mackey D (2016) DNest4: Diffusive Nested Sampling in C++ and Python URL http://arxiv.org/abs/1606.03757

Burke CJ, McCullough PR, Valenti Ja et al. (2007) XO2b: Transiting Hot Jupiter in a Metalrich Common Proper Motion Binary. The Astrophysical Journal 671(2):2115-2128, URL http: //stacks.iop.org/0004-637X/671/i=2/a=2115

Carter Ja Winn JN (2009) PARAMETER ESTIMATION FROM TIME-SERIES DATA WITH CORRELATED ERRORS: A WAVELET-BASED METHOD AND ITS APPLICATION TO TRANSIT LIGHT CURVES. ApJ 704(1):51-67, URL http://stacks.iop.org/0004-637X/704/i=1/a=51?key=crossref. 38a235bbafd054bbdf05d00d5c364393

Carter JA, Yee JC, Eastman J, Gaudi BS Winn JN (2008) Analytic Approximations for Transit LightCurve Observables, Uncertainties, and Covariances. ApJ 689(1):499-512, URL http: //adsabs.harvard.edu/abs/2008ApJ...689..499C

Carter JA, Winn JN, Gilliland R Holman MJ (2009) NEAR-INFRARED TRANSIT PHOTOMETRY OF THE EXOPLANET HD 149026b. The Astrophysical Journal 696(1):241-253, URL http://arxiv.org/abs/0902.1542

Chopin N Robert CP (2010) Properties of nested sampling. Biometrika 97(3):741-755, URL http://biomet.oxfordjournals.org/cgi/doi/10.1093/biomet/asq021

Claret A (2004) A new non-linear limb-darkening law for LTE stellar atmosphere models. III- Sloan filters: Calculations for-5. 0 not more than $\log$ not more than+ $1,2000 \mathrm{~K}$ not. A\&A 1005:1001-1005, URL http://www.csa.com/partners/viewrecord.php? requester=gs\&amp; collect ion=TRD\&amp; recid=2005091623165SO

Claret A (2008) Testing the limb-darkening coefficients measured from eclipsing binaries. A\&A 482(1):259-266, URL http://www. aanda.org/10.1051/0004-6361: 200809370

Claret A (2009) Does the HD 209458 planetary system pose a challenge to the stellar atmosphere models? A\&A 506(3):1335-1340, URL http://www . aanda.org/10.1051/ $0004-6361 / 200912423$ 
Claret A Bloemen S (2011) Gravity and limb-darkening coefficients for the Kepler, CoRoT, Spitzer, uvby, UBVRIJHK, and Sloan photometric systems. A\&A 529:A75, URL http: //www.aanda.org/10.1051/0004-6361/201116451

Claret A, Hauschildt PH Witte S (2012) New limb-darkening coefficients for PHOENIX/1D model atmospheres. Astronomy \& Astrophysics 546:A14, URL http: //www . aanda . org/10. 1051/0004-6361/201219849

Claret A, Dragomir D Matthews JM (2014) Theoretical gravity and limb-darkening coefficients for the MOST satellite photometric system. A\&A 567:A3, URL http://www. aanda.org/ articles/aa/full_html/2014/07/aa23515-14/aa23515-14.html

Clyde MA, Berger JO, Bullard F et al. (2007) Current Challenges in Bayesian Model Choice. In: Statistical Challenges in Modern Astronomy IV, vol 371, pp 224-240

Csizmadia S, Pasternacki T, Dreyer C et al. (2013) The effect of stellar limb darkening values on the accuracy of the planet radii derived from photometric transit observations. A\&A 549:A9, URL http://www.aanda.org/10.1051/0004-6361/201219888

Czekala I, Mandel KS, Andrews SM et al. (2017) Disentangling Time Series Spectra with Gaussian Processes: Applications to Radial Velocity Analysis URL http://arxiv.org/abs / 1702.05652

Espinoza N Jordan A (2015) Limb darkening and exoplanets: testing stellar model atmospheres and identifying biases in transit parameters. MNRAS 450(2):1879-1899, URL http://mnras . oxfordjournals.org/cgi/doi/10.1093/mnras/stv744

Feroz F, Hobson MP Bridges M (2009) MultiNest: an efficient and robust Bayesian inference tool for cosmology and particle physics. Monthly Notices of the Royal Astronomical Society 398(4):1601-1614, URL/https://academic.oup.com/mnras/article-lookup/ doi/10.1111/j.1365-2966.2009.14548.x

Feroz F, Hobson MP, Cameron E Pettitt AN (2013) Importance Nested Sampling and the MultiNest Algorithm pp 1-28, URL http: //arxiv.org/abs/1306.2144

Ford EB (2005) Quantifying the Uncertainty in the Orbits of Extrasolar Planets. AJ 129(3):17061717, URL http://stacks.iop.org/1538-3881/129/i=3/a=1706

Ford EB (2006) Improving the Efficiency of Markov Chain Monte Carlo for Analyzing the Orbits of Extrasolar Planets. ApJ 642(1):505-522, URL http://stacks.iop.org/ $0004-637 x / 642 / i=1 / a=505$

Ford EB, Street G Gregory PC (2007) Bayesian Model Selection and Extrasolar Planet Detection. In: STATISTICAL CHALLENGES IN MODERN ASTRONOMY IV, vol 371, pp 189-205

Foreman-Mackey D, Hogg DW, Lang D Goodman J (2013) emcee : The MCMC Hammer. Publications of the Astronomical Society of the Pacific 125(925):306-312, URL http://arxiv.org/abs/1202.3665http://www.jstor.org/stable/ info/10.1086/670067

Foreman-Mackey D, Agol E, Angus R Ambikasaran S (2017) Fast and scalable Gaussian process modeling with applications to astronomical time series URL http://arxiv.org/abs/ 1703.09710

Gelman A Rubin D (1992) Inference from iterative simulation using multiple sequences. Statistical science 7(4):457472, URL http://www. jstor.org/stable/2246093

Gelman A, Roberts GO Gilks WR (1996) Efficient Metropolis Jumping Rules. Bayesian statistics 5:599-607

Gelman A, Carlin JB, Stern HS et al. (2013) Bayesian Data Analysis, Third Edition, 3rd edn. CRC Press, URL https://books.google.com.pa/books?id=eSHSBQAAQBAJ

Gibson N, Aigrain S, Roberts SJ et al. (2012) A Gaussian process framework for modelling instrumental systematics: application to transmission spectroscopy. Monthly Notices of the Royal Astronomical Society 419(3):2683-2694, URL http://doi.wiley.com/10. 1111/j.1365-2966.2011.19915.xhttp://mnras.oxfordjournals.org/ cgi/doi/10.1111/j.1365-2966.2011.19915.x

Giménez A (2006) Equations for the analysis of the light curves of extra-solar planetary transits. A\&A 450(3):1231-1237, URL http://adsabs . harvard. edu/abs/2006A\&A. . $.450 .1231 \mathrm{G}$ 
Goodman J Weare J (2010) Ensemble samplers with affine invariance. Communications in Applied Mathematics and Computational Science 5(1):65-80, URL http://pjm. math. berkeley.edu/camcos/2010/5-1/p04.xhtml

Gregory PC (2005) Bayesian Logical Data Analysis for the Physical Sciences. Cambridge University Press, Cambridge

Gunter T, Osborne MA, Garnett R, Hennig P Roberts SJ (2014) Sampling for Inference in Probabilistic Models with Fast Bayesian Quadrature. Advances in Neural Information Processing Systems pp 2789-2797, URL http://papers.nips.cc/paper/ 5483-sampling-for-inference-in-probabilistic-models-with-fast-bayesian-quadrature

Hastings WK (1970) Monte Carlo sampling methods using Markov chains and their applications. Biometrika 57(1):97-109, URL http://biomet.oxfordjournals. org/content/57/1/97.abstracthttps://academic.oup.com/biomet/ article-lookup/doi/10.1093/biomet/57.1.97

Hennig P, Osborne MA Girolami M (2015) Probabilistic numerics and uncertainty in computations. Proceedings Mathematical, physical, and engineering sciences / the Royal Society 471(2179):20150,142, URL http://rspa.royalsocietypublishing.org/ content/471/2179/20150142

Hoffman MD Gelman A (2011) The No-U-Turn Sampler: Adaptively Setting Path Lengths in Hamiltonian Monte Carlo (2008):30, URL/http: //arxiv.org/abs/1111.4246

Holman MJ, Winn JN, Latham DW et al. (2006) The Transit Light Curve Project. I. Four Consecutive Transits of the Exoplanet XO1b. ApJ 652(2):1715-1723, URL http: //stacks . iop. org/0004-637X/652/i=2/a=1715

Kass RE Raftery AE (1995) Bayes factors. Journal of the american statistical association 90(430):773-795, URL/http://www.jstor.org/stable/10.2307/2291091

Kipping DM (2010) Investigations of approximate expressions for the transit duration. MNRAS 407(1):301-313, URL http://arxiv.org/abs/1004.3819

Kipping DM (2013) Efficient, uninformative sampling of limb darkening coefficients for two-parameter laws. Monthly Notices of the Royal Astronomical Society 435(3):21522160, URL http://mnras.oxfordjournals.org/cgi/doi/10.1093/mnras/ stt1435http://arxiv.org/abs/1308.0009

Kipping DM (2014) Characterizing distant worlds with asterodensity profiling. Monthly Notices of the Royal Astronomical Society 440(3):2164-2184, URL http://arxiv.org/ abs/1311.1170http://dx.doi.org/10.1093/mnras/stu318https:// academic.oup.com/mnras/article-lookup/doi/10.1093/mnras/stu318

Kipping DM (2016) Efficient, uninformative sampling of limb-darkening coefficients for a three-parameter law. Monthly Notices of the Royal Astronomical Society 455(2):1680 1690, URL https://academic.oup.com/mnras/article-lookup/doi/10. $1093 / \mathrm{mnras} / \mathrm{stv} 2379$

Link WA Eaton MJ (2012) On thinning of chains in MCMC. Methods in Ecology and Evolution 3(1):112-115

MacKay DJ (2003) Information Theory, Inference, and Learning Algorithms, 7th edn. Cambridge University Press, DOI 10.1017/S026357470426043X, URL http: //www.cambridge.org/0521642981http://www.inference.phy.cam. ac.uk/mackay/itila/http://www.journals.cambridge.org/abstract_ S026357470426043Xhttp://www.ncbi.nlm.nih.gov/pubmed/13217055

Mandel KS Agol E (2002) Analytic Light Curves for Planetary Transit Searches. ApJ 580(2):L171-L175, URL http: //adsabs . harvard.edu/abs/2002ApJ . . 580L. $171 \mathrm{M}$

Matthews AGdG, van der Wilk M, Nickson T et al. (2016) GPflow: A Gaussian process library using TensorFlow pp 1-6, URL http: //arxiv.org/abs/1610.08733

Müller HM, Huber KF, Czesla S, Wolter U Schmitt JHMM (2013) High-precision stellar limbdarkening measurements. A\&A 560:A112, URL http://www.aanda.org/10.1051/ $0004-6361 / 201322079$ 
Osborne MA, Garnett R, Roberts SJ et al. (2012) Bayesian Quadrature for Ratios. Proceedings of the Fifteenth International Conference on Artificial Intelligence and Statistics 22:832-840, URL http://jmlr.csail.mit.edu/proceedings/papers/v22/osborne12. html

Parviainen H Aigrain S (2015) ldtk: Limb Darkening Toolkit. Monthly Notices of the Royal Astronomical Society 453(4):3821-3826, URL http://arxiv.org/ abs/1508.02634http://dx.doi.org/10.1093/mnras/stv1857http: //mnras.oxfordjournals.org/lookup/doi/10.1093/mnras/stv1857

Rajpaul V, Aigrain S, Osborne MA, Reece S Roberts SJ (2015) A Gaussian process framework for modelling stellar activity signals in radial velocity data. Monthly Notices of the Royal Astronomical Society 452(3):2269-2291

Rasmussen CE Ghahramani Z (2002) Bayesian monte carlo. Advances in neural ... (1), URL http://machinelearning.wustl.edu/mlpapers/paper_files/AA01.pdf

Rasmussen CE Williams C (2006) Gaussian processes for machine learning. The MIT Press, URL http://citeseerx.ist.psu.edu/viewdoc/summary?doi=10.1.1.86. 3414

Robert CP (2007) The Bayesian Choice. Springer, New York

Roberts GO, Gelman A Gilks WR (1997) Weak convergence and optimal scaling of random walk Metropolis algorithms. Annals of Applied Probability 7(1):110-120

Roberts SJ, Osborne MA, Ebden M et al. (2013) Gaussian processes for time-series modelling. Philosophical transactions Series A, Mathematical, physical, and engineering sciences 371(1984):20110,550, URL http://www.ncbi.nlm.nih.gov/pubmed/2327760 7

Salvatier J, Wiecki T Fonnesbeck C (2015) Probabilistic Programming in Python using PyMC. Arxiv pp 1-24, URL http://arxiv.org/abs/1507.08050

Seager S Mallen-Ornelas G (2003) A Unique Solution of Planet and Star Parameters from an Extrasolar Planet Transit Light Curve. ApJ 585(2):1038-1055, URL http://adsabs . harvard.edu/abs/2003ApJ...585.1038S

Shah A, Wilson AG Ghahramani Z (2014) Student-t Processes as Alternatives to Gaussian Processes 33:13, URL http: //arxiv.org/abs/1402.4306

Sing DK (2010) Stellar limb-darkening coefficients for CoRot and Kepler. A\&A 510:A21, URL http://www.aanda.org/10.1051/0004-6361/200913675

Sing DK, Désert JM, Lecavelier des Etangs A et al. (2009) Transit spectrophotometry of the exoplanet HD 189733b. Astronomy and Astrophysics 505(2):891-899, URL http://arxiv.org/abs/0907.4991v1\%5Cnpapers3://publication/ uuid/2E560D 9E-CF3E-44A1-B205-25C2C919A5F8http://www.aanda.org/ $10.1051 / 0004-6361 / 200912776$

Skilling J (2004) Nested Sampling. AIP Conference Proceedings 735:395-405, URL http:// link.aip.org/link/?APC/735/395/1\&Agg=doi

Skilling J (2006) Nested sampling for general Bayesian computation. In: ISBA 8th World Meeting on Bayesian Statistics, URL http://projecteuclid.org/euclid.ba/ 1340370944

Tingley B, Bonomo AS Deeg HJ (2011) USING STELLAR DENSITIES TO EVALUATE TRANSITING EXOPLANETARY CANDIDATES. ApJ 726(2):112, URL http://stack.s.iop.org/0004-637X/726/i=2/a=112?key=crossref. 1f7718777e8ac3923c916ecf58d816a4

Tran D, Kucukelbir A, Dieng AB et al. (2016) Edward: A library for probabilistic modeling, inference, and criticism URL http://arxiv.org/abs/1610.09787 